1

\title{
Behaviour of plastic litter in nearshore waters: first insights from wind and wave laboratory experiments
}

\author{
Pernille Louise Forsberg ${ }^{(a)}$, Damien Sous ${ }^{(a, b)}$, Alessandro Stocchino ${ }^{(c)}$, Remi Chemin ${ }^{(b)}$ \\ (a) Univ Pau \&S Pays Adour / E2S UPPA, Chaire HPC-Waves, Laboratoire des Sciences de l'Ingénieur Appliquées \\ à la Méchanique et au Génie Electrique - Fédération IPRA, EA4581, 64600, ANGLET, France \\ (b) Université de Toulon, Aix Marseille Université, CNRS, IRD, Mediterranean Institute of Oceanography (MIO), \\ La Garde, France \\ (c) Department of Civil, Chemical and Environmental Engineering, University of Genoa, Italy, Via Montallegro 1 , \\ Genoa, 16145, Italy
}

\begin{abstract}
Plastic litter in nearshore waters is an environmental pollutant with increasing impact on coastal environments. At present, knowledge on basic plastic particle dynamics and the interaction with complex hydrodynamics is lacking. The present laboratory study, performed under controlled wave and wind conditions, demonstrates the dispersion of plastics in shallow waters. The study presents a simple case looking solely at cross-shore particle transport. The results show that both wind and waves as well as plastic properties (shape and density) govern the behaviour of plastic litter in the nearshore zone. Heavy particles behave like natural sand with accumulation in the wave breaking zone. Light particles have varying accumulation along the coastal profile depending on the wave and wind field and the particle shape. More extensive characterization remains to be done in future studies.
\end{abstract}

Keywords: plastic; pollution; nearshore zone; waves; wind. 


\section{Introduction}

Plastic pollution is a major hazard to coastal ecosystems. The release of plastic to the ocean cause long term challenges for the sustainability of marine environments due to the resistance of plastic to biodegrade [1]. Plastic has been found dispersed in the open ocean and nearshore areas, deposited in sedimentary habitats and incorporated into food webs through ingestion by marine organisms $[2,3,4,5]$. Several reviews concerning plastic pollution in the marine environment have been carried out, e.g. $[6,7,8,9,10,11,12]$. The primary focus has been on the global distribution of plastic, the impact of plastic on the environment and living organisms, and societal recommendations. Fundamental questions still remain unresolved concerning, for instance, the fate and transport of plastic litter in shallow waters [13, 14, 15].

Plastic is comprised of a long chain of polymers. Typical polymers that are found in oceans include polypropylene and polyethylene [16] as well as polyvinyl chloride and polyamide [17]. Plastics have different physical properties in terms of density, shape and size, and are typically size-divided into macro- $(\geq 25 \mathrm{~mm})$, meso- $(<25 \mathrm{~mm}-5 \mathrm{~mm})$, micro- $(<5 \mathrm{~mm}-1 \mathrm{~mm})$, mini-micro- $(<1 \mathrm{~mm}-1$ $\mu \mathrm{m})$ and nanoplastics $(<1 \mu \mathrm{m})[5]$. The diverse physical properties of plastics impact the behavior and fate of the particles in the marine environment [18]. Plastic debris is usually considered as passive tracers; i.e. they do not influence the surrounding water properties, but evolve under the action of chemical processes such as degradation and biofouling, or physical processes, which will modify their shape, size and density [19].

The main driver of plastic dispersion in the deep ocean is currents acting on a wide range of spatio-temporal scales: basin-scale currents, tidal currents, upwelling, convergence zones, etc. Wind also has a major effect on plastic dispersion, first by applying a direct drag force on the floating and emerged parts of the plastic debris and indirectly by driving surface currents and increasing mixing and shear in the water column [20]. Waves are also known to transport matter via Stoke's drift and roller advection under breaking wave conditions [21]. Forced by a combination of shear stress, wind and breaking waves, turbulence increases the mixing and diffusion of plastic particles. In addition to this forcing, buoyancy is also of primary importance, depending both on the plastic density and the surrounding density field, which may be stratified. Note that plastic density is expected to evolve due to degradation and biofouling [22, 23].

Approaching nearshore waters, the physics of plastic dispersion is expected to be further complicated by the effect of shallowing bathymetry, which is responsible for strong modifications of the wave field (shoaling and breaking) and the related generation of currents. So far, plastic behaviour in nearshore waters is much less documented than in open oceans [24]. Nearshore zones are often considered a temporary sink for plastics due to the nearshore hydrodynamics forcing onshore transport $[25,26,14]$. However, nearshore zones can also be a source for plastics to the open ocean [3], especially during extreme events [27].

A conceptual basis to understand and predict plastic behavior in nearshore areas is through sediment transport. The general understanding of cross-shore sediment transport is that the motion of sediment is driven by the competition of return 'undertow' flow near the middle of the water column and the effect of wave skewness and asymmetry near the bottom [21]. The imbalance between these processes leads to the classical hypothesis of onshore/offshore transport under moderate and stormy wave conditions. However, the analogy with plastic debris is not straightforward because plastics generally are much lighter than natural sediment. The density of plastic debris typically range in the order of 0.9-1.4 $\mathrm{g} \mathrm{cm}^{-3}$, while the density of common coastal sediment is $2.65 \mathrm{~g} \mathrm{~cm}^{-3}$ (quartz). Plastics will therefore respond differently to undertow and wave asymmetry effects, and will potentially be more driven by surface processes such as stokes drift, roller advection and wave-driven turbulence. The wind is again expected to play an additional role to the wave-induced transport. The importance of all these processes is dependent on the density and the size of the plastic particles and, thus, by the corresponding inertial effects in terms of drag force and buoyancy.

Overall, the wind-, wave- and flow-driven transport mechanisms are expected to dominate specific compartments of the water column, inducing a differential distribution of plastic particles. Entering nearshore areas, the shallowing bathymetry will lead to a general increase in the effect of each of these forces, and a potential collapse of the wind-, wave-, and flow-driven layers. 
Understanding the behaviour of plastic particles in the nearshore zone is not trivial and involves a range of processes which are not well understood at present. The experiments in the present study were performed in order to obtain a first insight into the transport of plastic particles in the nearshore zone. The study presents a simple case looking solely at cross-shore particle transport and the effect of wind and waves on the behaviour of plastics. The specific objectives are to quantify how shallow plastic litter distribution depends on the plastic properties (shape and density) as well as on the wave and wind features.

\section{Laboratory experiments}

\subsection{Experimental setup}

The experiments were carried out in the CASH (Canal Aero-Sedimento-Hydrodynamics) windwave flume at the Seatech engineering school, University of Toulon. The flume has a length of 6 $\mathrm{m}$ and a width of $0.5 \mathrm{~m}$ and was equipped with a linearly sloping bathymetry (figure 1). The still water depth was $0.22 \mathrm{~m}$ at the deep end of the tank, here regular waves were produced by a piston wave maker. On- and offshore wind conditions were produced by a closed circuit, reversible wind blower. The wind speed was set to $6 \mathrm{~m} \mathrm{~s}^{-1}$ in both directions. The wave features were investigated with nine wave gauges measuring water level variations with a sampling frequency of $256 \mathrm{~Hz}$. The wave gauges were evenly distributed in the flume with an internal distance of $0.3 \mathrm{~m}$. The crossshore flow circulation was investigated with two side-looking acoustic doppler velocimeters (ADVs, Nortek Vectrinos). The ADVs measured surface and near-bed current direction and magnitude at a $200 \mathrm{~Hz}$ sampling frequency in the shoaling zone and near the breaking point. Note that the surface measurements were carried out near the middle of the water column, due to the necessity of keeping the instruments permanently immersed even under wave troughs. A range of contrasted plastic particle shapes and densities were selected to represent the diversity of plastics found in coastal waters. Three typical shapes were used: nearly spherical pellets about $3 \mathrm{~mm}$ in diameter, squared sheets with $5 \mathrm{~mm}$ sides and fibres of $5 \mathrm{~mm}$ length. The plastic pellets were bought from a manufacturer, while the plastic sheets and fibres were made from everyday materials such as plastic file folders and broom hair. Four types of plastics were used: low density polyethylene $\left(0.92 \mathrm{~g} \mathrm{~cm}^{-3}\right.$, transparent pellets and yellow sheets), polypropylene $\left(0.95 \mathrm{~g} \mathrm{~cm}^{-3}\right.$, yellow fibres), polyamide (1.15 $\mathrm{g} \mathrm{cm}^{-3}$, green fibres) and polyvinyl chloride $\left(1.38 \mathrm{~g} \mathrm{~cm}^{-3}\right.$, white pellets and red sheets). The water in the tank was freshwater with a density of about $1 \mathrm{~g} \mathrm{~cm}^{-3}$. The plastic particles were categorized as either light or heavy based on their densities relative to the water density.
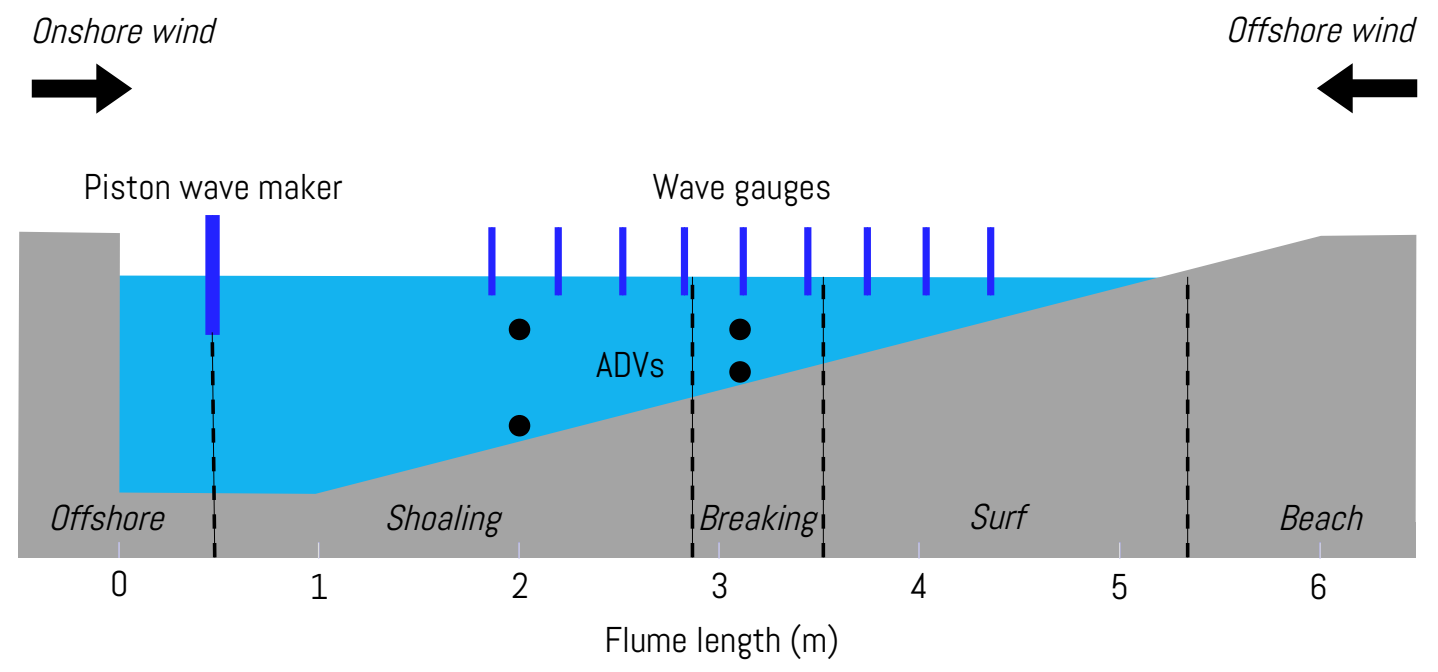

Figure 1: Experimental setup; wind forcing was generated in a closed $10 \mathrm{~m}$ long circuit by a reversible wind blower. 


\subsection{Procedure}

A reference wave case consisting of regular waves (wave height and period of $9.2 \mathrm{~cm}$ and 1.2 $\mathrm{s}$, respectively) was run with full instrumentation without plastic particles in order to obtain a complete view of the cross-shore evolution of wave height and currents during no-wind, onshore and offshore wind conditions. An additional wave case in no-wind conditions was performed with a wave height and period of $8 \mathrm{~cm}$ and $0.7 \mathrm{~s}$, respectively.

Each wave and wind case was run with plastic particles without instruments in order to minimize disturbance within the flume. Fifty plastic particles of each type (350 in total) were released simultaneously into the active flume in the beginning of the shoaling zone at a depth of $-0.1 \mathrm{~m}$. The amount of time it took for the particles to reach the beach following release, i.e. the beaching time, was visually tracked. The beaching time was noted for all particles except for light pellets. The light pellets were transparent and could therefore not be clearly tracked. Each experiment ran for at least five minutes until a stationary state was reached; i.e. when no significant motion of plastic particles was observed anymore. Immediately after the wave maker was shut off, the flume was manually compartmentalized into five zones (offshore, shoaling, breaking, surf and beach zone) using wood panels to avoid spurious particle migration. The plastic particles were all carefully recovered from each compartment and counted.

\subsection{Data processing}

The wave height was estimated based on peak analysis of the water level fluctuations captured by the wave gauges. The cross-shore position of the wave breaking zone was visually identified from the start of the wave overturning phase up to the end of the splash-up process where the fully saturated surf zone started. The current speed and direction was estimated based on the wave-run averaged horizontal velocity component.

\section{Results}

\subsection{Hydrodynamic conditions}

The wave breaking point for the reference case was located at the inflexion of the cross-shore wave height profile; i.e. approximately at $\mathrm{x}=3.5 \mathrm{~m}$ (figure 2). The surface ADV showed the typical return flow observed in breaking and shoaling zones [28, 29]. The bottom ADV showed a weak return flow in the breaking zone and very small flow with an onshore direction in the shoaling zone. This probably indicated the presence of a weak deep water circulation forced by the presence of a closed boundary. The main effects of offshore wind was (i) increased return flow measured at the surface and (ii) promoted wave plunging and slightly later breaking. The main effects of onshore wind was (i) increased return flow at the bed in the breaking zone, probably revealing the compensation of stronger onshore transport in the surface layer, and (ii) earlier and smoother spilling type breaking. The main effects of shorter waves was (i) slightly smaller wave heights in the shoaling zone, but similar wave heights compared to the reference case in the surf zone, and (ii) a weaker return current in the breaking zone. Current information was not available for shorter waves in the shoaling zone. 

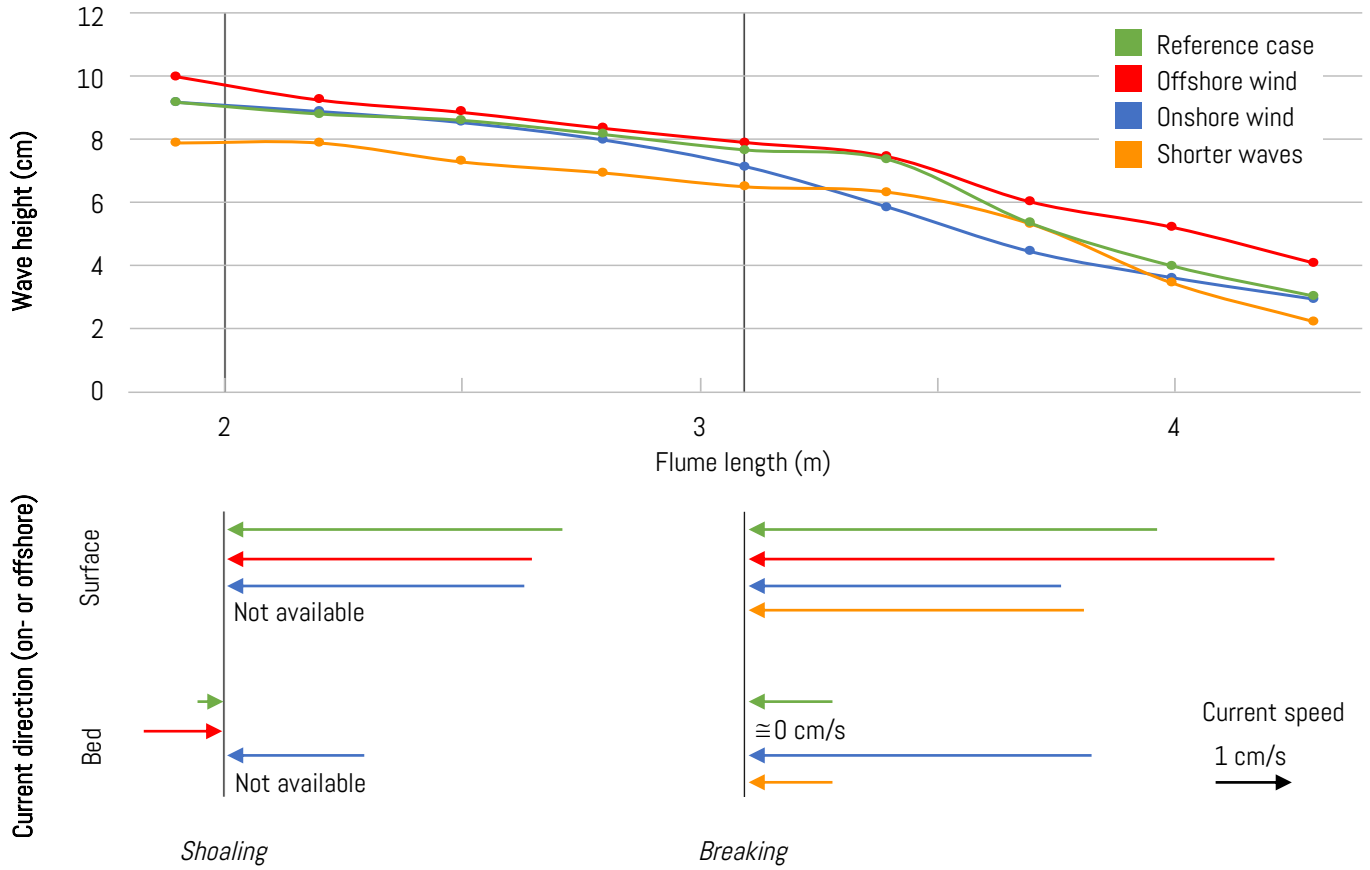

Flume length $(\mathrm{m})$

Figure 2: Top: Cross-shore evolution of wave height in the shoaling and breaking zone. Bottom: Current direction at the location of the two ADVs in the shoaling and breaking zone; the arrows indicate mean current magnitude and direction in an onshore and offshore direction.

\subsection{Plastic distribution and dynamics}

The overall trend for the cross-shore distribution of plastic particles is straightforward (figure 3). In the reference case, nearly all heavy plastic particles (polyamide, polyvinyl chloride) are trapped in the breaking zone. They rapidly dropped to the bed after release and progressively moved onshore due to wave non-linearity in the shoaling zone. Reaching the breaking zone, they were maintained in a nearly stationary position by the local balance between return flow and wave forcing. Here there is a direct similarity to natural sand motion forming sand bars under the breaking point. Lighter particles (low density polyethylene, polypropylene) initially followed the same onshore motion after release. Near the breaking point, most of them were rapidly caught and advected by the roller across the surf zone. The final distribution was partly dependent on the particle shape. Most of the sheets were driven offshore by the return flow and remained trapped in the surf zone. Round particles (pellets) beached once they reached the swash zone.

For the offshore wind case, the distribution of heavy plastic particles followed the same pattern as in the reference case, the only difference being the onshore shift of the breaking zone. The distribution of light plastic particles was dramatically different compared to the reference case. The light plastic particles moved offshore immediately after release due to the surface stress applied by the offshore wind. The particles were not able to reach the breaking zone. The effect of plastic particle shape was here negligible. The onshore wind case appeared to be very similar to the reference case with heavy plastic particles being trapped in the breaking zone and light plastic particles being trapped in the surf zone or on the beach. The main difference was the slight offshore shift of the breaking zone due to the effect of onshore wind.

The short wave case showed some difference compared to the reference case, in particular for the heavy particles. Approximately one third of the heavy particles were stuck in the shoaling zone. This could be related to the smaller effect of wave non-linearity for shorter waves. 


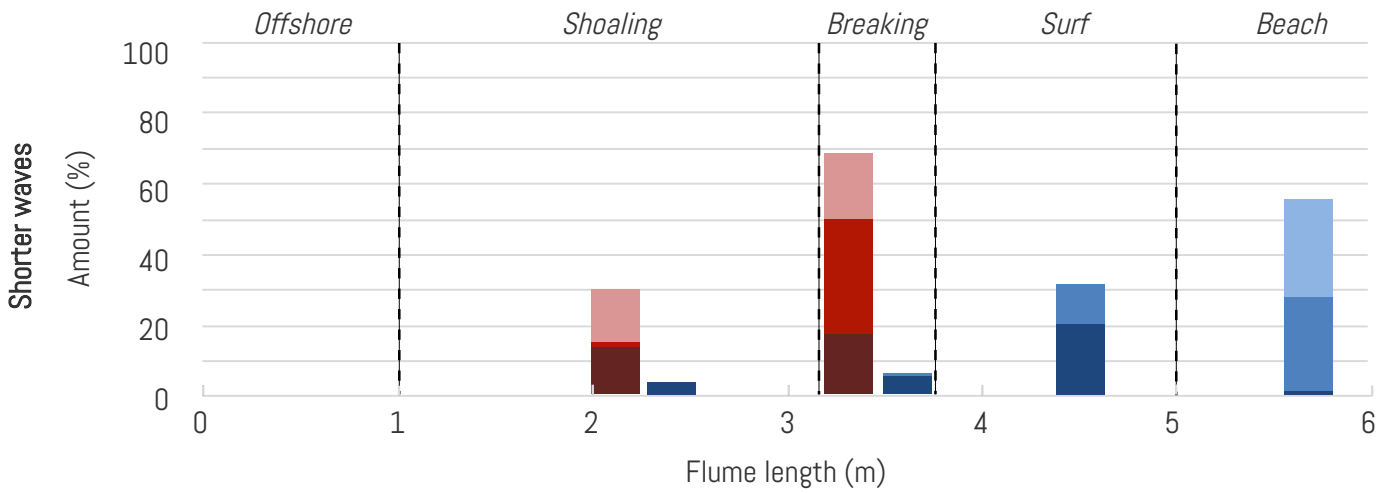

Figure 3: Cross-shore distribution of plastic particles for the four wave cases and indications of the location of the offshore, shoaling, breaking, surf and beach zone.

The beaching time for the plastic particles varied according to the plastic density and shape as well as to the wind and wave conditions. Recall, it was not possible to track the light pellets properly due to their transparency, thus they are not included in the study of beaching times. On average, it took $165 \mathrm{~s}$ for light fibres and $300 \mathrm{~s}$ for light sheets to beach during the reference wave 
case. No particles beached during offshore wind conditions. During onshore wind conditions it took $150 \mathrm{~s}$ for light fibres and $290 \mathrm{~s}$ for light sheets to beach. For the short wave case it took $105 \mathrm{~s}$ for light fibres and $215 \mathrm{~s}$ for light sheets to beach.

\section{Discussion and Conclusions}

Plastic particle behaviour in shallow water is important for model studies of plastic distribution in coastal areas [30, 14]. Major limitations are present in most model studies due to a lack of knowledge on basic plastic particle dynamics and the interaction with complex hydrodynamics $[16,18,31,32,33]$. It has previously been suggested that waves in combination with wind, currents and plastic densities affect the accumulation patterns of plastic litter on beaches $[17,34,35,36]$. However, a basic assumption is that beaches are a sink of plastic particles owing to onshore transport by waves $[25,26,14]$.

The present study, performed under controlled wave and wind conditions in the laboratory, demonstrates vividly that dispersion of plastics in the nearshore zone cannot be summed up as a bulk onshore motion, but that it involves a series of fine interactions among several physical processes and the particle features [33]. The most illustrative case is the one for heavy particles.

Heavy plastic particles are expected to behave like natural sand with accumulation in the breaking zone near the bed or mixed with natural sediment. One should therefore expect to find important plastic contamination in this area. The strong similarity between heavy plastic particles and sediment suggests that inertial effects and the interaction with the hydrodynamic forcing (wake drift, wind shear) is crucial. The final journey of a given plastic particle thus also results from the dynamic balance between wind-, wave- and flow-driven transport. These transport modes act differently in the nearshore zone with varying magnitudes both vertically in the water column and horizontally along the shoaling and surf zone. The cross-shore dispersion of heavy sheets highlights the sensitivity of the net transport to the spatial variations in wind-, wave- and flow-driven transport. Near the surface or the bottom, the transport is generally dominated by a single transport mechanism, leading to a clear final cross-shore distribution of particles at the end of the experiment. For particles residing in the middle of the water column, such as heavy sheets, a slight vertical displacement can bring the particles from a layer controlled by a given dynamic balance to another, potentially dominated by a different transport mechanism. This sensitivity of the particle position along its track, which may be compared to deterministic chaos system uncertainty, induces a higher spread in final the cross-shore distribution.

The cross-shore distribution of light plastic particles is controlled by both the wave- and wind conditions as well as the particle shapes. Offshore wind is expected to flush light plastic particles away from shore. In no-wind or onshore winds conditions, light plastic particles entering the breaking zone are rapidly advected towards the shore by the waves, like surfers. Plastic particles that have a shape with a small drag, such as pellets (i.e. high settling velocity) are prone to remain in the swash zone and finally be deposited on the beach. Plastic particles with a high drag, such as sheets (i.e. low settling velocity) are more sensitive to the return flow. The majority of these particles remained in the surf zone driven by the competition between return flow, wave non-linearity and roller advection. The average beaching time for the light sheets that finally did deposit on the beach was two times faster than the beaching time for light fibres.

An important factor for nearshore and coastal systems, which has been ignored here, is the water salinity. The present experiments only addressed freshwater conditions. Increasing the water salinity will necessarily lead to an increase in water density and a related increase in buoyancy forces. Such effects should be accounted for in particular for plastic densities ranging between fresh- and saltwater densities (i.e. between 1.0 and about $1.035 \mathrm{~g} \mathrm{~cm}^{-3}$ ). An increase in water density could cause a shift in the particle behaviour from the heavy to the light regimes observed here, if the water density exceeds the particle density. An increase in water density could therefore have a significant effect on the particle dynamics, with an expected overall upward shift of the particle load, promoting surface-related processes. These conceptual assumptions remain to be validated in the field with a more global assessment of the particle dispersion. However, the main trends 
observed here should remain valid as soon as the heavy versus light discrimination is carried out relative to the effective water density considered at a given site.

The present study demonstrates the behaviour of plastic particles in nearshore waters looking solely at cross-shore transport. While the basic mechanisms summarized in the study should remain valid in nature, a number of limitations and unaddressed issues require further investigations. The first main issue is scaling effects. When performing laboratory experiments challenges related to dimensional scaling are almost inevitable. For the present study, the aim was to get a first insight into plastic particle behaviour in the nearshore zone, and to understand the underlying physical processes. For this reason, potential scaling effects were not considered. Further limitations to the study include the impact of longshore currents, which was neglected. Longshore currents could both add and remove plastic particles to and from the system. Another limitation is the linear bed, which did not account for features such as bars, troughs and ripples that are typically found on sandy shorefaces. Realistic bathymetric features would have an impact on the wave and current field and therefore on the plastic particle distribution. Using regular waves simplified the irregular nature of waves in the field and thus served to maintain a simple analysis. The role of wave types, however, clearly deserves further investigation, especially the role played by long infragravity waves [37]. Plastic particle properties, i.e. density and shape, showed to play an important role for the particle distribution. Therefore, a more extensive characterization remains to be done. To sum up, it involves a wide range of processes to understand the fate of plastics in nearshore environments. The first insights obtained in the present paper showed that both wind and waves as well as plastic properties governed the behaviour of plastic litter in nearshore waters.

\section{Acknowledgements}

The authors would like to thank Aimed Ajroud for his help during the laboratory experiments. This research has been financially supported by the Project Splash!, Stop alle Plastiche in H20!, funded by the Interreg Italia-Francia Marittimo Program. Additional support has been provided by the MIDYNET project (CNRS MITI). 


\section{References}

[1] T. C. Jennerjahn, S. B. Mitchell, Pressures, stresses, shocks and trends in estuarine ecosystems - an introduction and synthesis, Estuarine, Coastal and Shelf Science 130 (2013) 1-8 (2013).

[2] R. C. Thompson, Y. Olsen, R. P. Mitchell, A. Davis, S. J. Rowland, A. W. John, D. McGonigle, A. E. Russell, Lost at sea: where is all the plastic?, Science 304 (5672) (2004) 838-838 (2004).

[3] E. van Sebille, M. H. England, G. Froyland, Origin, dynamics and evolution of ocean garbage patches from observed surface drifters, Environmental Research Letters 7 (4) (2012) 044040 (2012).

[4] D. Cressey, Bottles, bags, ropes and toothbrushes: the struggle to track ocean plastics, Nature News 536 (7616) (2016) 263 (2016).

[5] C. Crawford, B. Quinn, Microplastics, standardisation and spatial distribution, in: Microplastic Pollutants, Elsevier, 2017, Ch. 5, pp. 101-130 (2017).

[6] J. A. I. do Sul, M. F. Costa, The present and future of microplastic pollution in the marine environment, Environmental pollution 185 (2014) 352-364 (2014).

[7] S. C. Gall, R. C. Thompson, The impact of debris on marine life, Marine pollution bulletin 92 (1-2) (2015) 170-179 (2015).

[8] L. G. A. Barboza, B. C. G. Gimenez, Microplastics in the marine environment: current trends and future perspectives, Marine pollution bulletin 97 (1-2) (2015) 5-12 (2015).

[9] J. Wang, Z. Tan, J. Peng, Q. Qiu, M. Li, The behaviors of microplastics in the marine environment, Marine Environmental Research 113 (2016) 7-17 (2016).

[10] D. Xanthos, T. R. Walker, International policies to reduce plastic marine pollution from singleuse plastics (plastic bags and microbeads): a review, Marine pollution bulletin 118 (1-2) (2017) $17-26$ (2017).

[11] K. L. Law, Plastics in the marine environment, Annual review of marine science 9 (2017) 205-229 (2017).

[12] S. Rezania, J. Park, M. F. M. Din, S. M. Taib, A. Talaiekhozani, K. K. Yadav, H. Kamyab, Microplastics pollution in different aquatic environments and biota: A review of recent studies, Marine pollution bulletin 133 (2018) 191-208 (2018).

[13] M. Cole, P. Lindeque, C. Halsband, T. S. Galloway, Microplastics as contaminants in the marine environment: a review, Marine pollution bulletin 62 (12) (2011) 2588-2597 (2011).

[14] H. Zhang, Transport of microplastics in coastal seas, Estuarine, Coastal and Shelf Science 199 (2017) 74-86 (2017).

[15] I. Chubarenko, E. Esiukova, A. Bagaev, I. Isachenko, N. Demchenko, M. Zobkov, I. Efimova, M. Bagaeva, L. Khatmullina, Behavior of microplastics in coastal zones, in: Microplastic Contamination in Aquatic Environments, Elsevier, 2018, Ch. 6, pp. 175-223 (2018).

[16] A. Isobe, K. Kubo, Y. Tamura, E. Nakashima, N. Fujii, et al., Selective transport of microplastics and mesoplastics by drifting in coastal waters, Marine pollution bulletin 89 (1-2) (2014) $324-330$ (2014).

[17] M. A. Browne, T. S. Galloway, R. C. Thompson, Spatial patterns of plastic debris along estuarine shorelines, Environmental Science \& Technology 44 (9) (2010) 3404-3409 (2010).

[18] I. Chubarenko, A. Bagaev, M. Zobkov, E. Esiukova, On some physical and dynamical properties of microplastic particles in marine environment, Marine pollution bulletin 108 (1-2) (2016) 105-112 (2016). 
[19] A. L. Andrady, Microplastics in the marine environment, Marine pollution bulletin 62 (8) (2011) 1596-1605 (2011).

[20] T. Kukulka, G. Proskurowski, S. Morét-Ferguson, D. Meyer, K. Law, The effect of wind mixing on the vertical distribution of buoyant plastic debris, Geophysical Research Letters 39 (7) (2012).

[21] F. Grasso, H. Michallet, E. Barthélemy, Sediment transport associated with morphological beach changes forced by irregular asymmetric, skewed waves, Journal of Geophysical Research: Oceans 116 (2011).

[22] A. Porter, B. P. Lyons, T. S. Galloway, C. Lewis, Role of marine snows in microplastic fate and bioavailability, Environmental science \& technology 52 (12) (2018) 7111-7119 (2018).

[23] M. Kooi, E. H. v. Nes, M. Scheffer, A. A. Koelmans, Ups and downs in the ocean: effects of biofouling on vertical transport of microplastics, Environmental science \& technology 51 (14) (2017) 7963-7971 (2017).

[24] M. H. DiBenedetto, N. T. Ouellette, J. R. Koseff, Transport of anisotropic particles under waves, Journal of Fluid Mechanics 837 (2018) 320-340 (2018).

[25] M. A. Browne, P. Crump, S. J. Niven, E. Teuten, A. Tonkin, T. Galloway, R. Thompson, Accumulation of microplastic on shorelines worldwide: sources and sinks, Environmental science \& technology 45 (21) (2011) 9175-9179 (2011).

[26] M. Thiel, I. Hinojosa, L. Miranda, J. Pantoja, M. Rivadeneira, N. Vásquez, Anthropogenic marine debris in the coastal environment: A multi-year comparison between coastal waters and local shores, Marine pollution bulletin 71 (1-2) (2013) 307-316 (2013).

[27] L. C.-M. Lebreton, J. C. Borrero, Modeling the transport and accumulation floating debris generated by the 11 march 2011 tohoku tsunami, Marine pollution bulletin 66 (1-2) (2013) $53-58(2013)$.

[28] U. Putrevu, I. A. Svendsen, Vertical structure of the undertow outside the surf zone, Journal of Geophysical Research: Oceans 98 (C12) (1993) 22707-22716 (1993).

[29] A. Reniers, E. Thornton, T. Stanton, J. Roelvink, Vertical flow structure during sandy duck: observations and modeling, Coastal Engineering 51 (3) (2004) 237-260 (2004).

[30] K. Critchell, J. Lambrechts, Modelling accumulation of marine plastics in the coastal zone; what are the dominant physical processes?, Estuarine, Coastal and Shelf Science 171 (2016) $111-122(2016)$.

[31] S. Iwasaki, A. Isobe, S. Kako, K. Uchida, T. Tokai, Fate of microplastics and mesoplastics carried by surface currents and wind waves: A numerical model approach in the sea of japan, Marine pollution bulletin 121 (1-2) (2017) 85-96 (2017).

[32] A. Bagaev, A. Mizyuk, L. Khatmullina, I. Isachenko, I. Chubarenko, Anthropogenic fibres in the baltic sea water column: Field data, laboratory and numerical testing of their motion, Science of The Total Environment 599 (2017) 560-571 (2017).

[33] I. Jalón-Rojas, X. H. Wang, E. Fredj, A 3d numerical model to track marine plastic debris (trackmpd): Sensitivity of microplastic trajectories and fates to particle dynamical properties and physical processes, Marine Pollution Bulletin 141 (2019) 256-272 (2019).

[34] C. Eriksson, H. Burton, S. Fitch, M. Schulz, J. van den Hoff, Daily accumulation rates of marine debris on sub-antarctic island beaches, Marine pollution bulletin 66 (1-2) (2013) 199 208 (2013). 
[35] I.-S. Kim, D.-H. Chae, S.-K. Kim, S. Choi, S.-B. Woo, Factors influencing the spatial variation of microplastics on high-tidal coastal beaches in korea, Archives of environmental contamination and toxicology 69 (3) (2015) 299-309 (2015).

[36] N. H. E. Ho, C. Not, Selective accumulation of plastic debris at the breaking wave area of coastal waters, Environmental Pollution 245 (2019) 702-710 (2019).

[37] X. Bertin, A. de Bakker, A. Van Dongeren, G. Coco, G. Andre, F. Ardhuin, P. Bonneton, F. Bouchette, B. Castelle, W. C. Crawford, et al., Infragravity waves: From driving mechanisms to impacts, Earth-Science Reviews 177 (2018) 774-799 (2018). 


\section{${ }^{*}$ Declaration of Interest Statement}

\section{Declaration of interests}

$\bigotimes$ The authors declare that they have no known competing financial interests or personal relationships that could have appeared to influence the work reported in this paper.

$\square$ The authors declare the following financial interests/personal relationships which may be considered as potential competing interests:

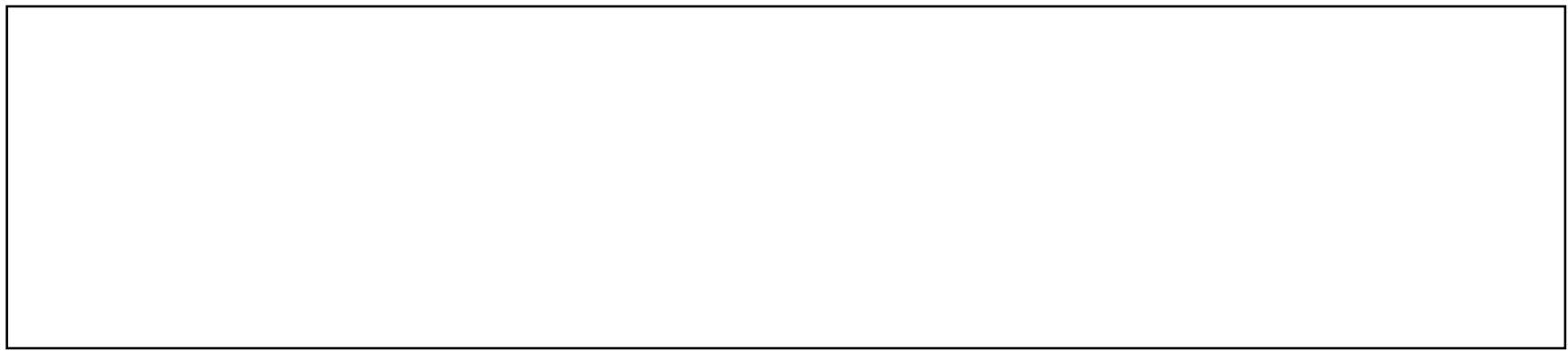


Credit Author Statement

PL. Forsberg: Conceptualization; Data curation; Formal analysis; Investigation; Methodology; Roles/Writing - original draft; Writing - review \& editing.

D. Sous: Conceptualization; Data curation; Formal analysis; Funding acquisition; Investigation; Methodology; Roles/Writing - original draft; Writing - review \& editing.

A. Stocchino: Conceptualization; Formal analysis; Investigation; Writing - review \& editing.

R. Chemin: Data curation; Formal analysis; Investigation; Methodology; Writing - review \& editing. 